\title{
Diagnosis of sarcoidosis - the updated ATS 2020 recommendations through the prism of everyday clinical practice
}

\section{Introduction}

Sarcoidosis is a chronic multiorgan granulomatous disease of unknown etiology, which most often involves the lungs and intrathoracic lymph nodes [1]. The latest international guidelines for diagnosis and management of the disease were published in 1999 [1]. An update of the official position of the American Thoracic Society (ATS) expert regarding the Clinical Practice Guideline for the Diagnosis of Sarcoidosis was released in April, this year [2].

The document does not present a significantly changed approach to the diagnosis of the disease but presents an expert position on certain clinical situations that the practitioner meets when determining the diagnosis of sarcoidosis. The guidelines were developed according to the GRADE methodology (Grading of Recommendations Assessment, Development, and Evaluation), based on a systematic review of the literature and, where appropriate, meta-analysis, in order to summarize the best available evidence.

Diagnosis of sarcoidosis is not standardized, but there is a general consensus that it should be based on the following criteria: consistent, adequate clinical presentation, demonstration of the presence of granulomatous lesions in pathomorphological examination (in one or more tissue samples) and the exclusion of alternative causes of granulomatous disease [2].

In this article, the reader will find, briefly presented, the most important position of ATS experts regarding selected aspects of the diagnosis of sarcoidosis, with a comment from the authors of this editorial.

\section{Summary of the ATS 2020 recommendations}

\section{Pathomorphological examination of lymph nodes}

In patients who are highly likely to have a clinical diagnosis of sarcoidosis (e.g. Lofgren's syndrome, symptoms of "lupus pernio" or Heerfordt's syndrome), a panel of experts allows (suggests) NOT sampling lymph nodes (conditional recommendation, very low quality evidence). In asymptomatic patients with changes in the radiological image indicating bilateral symmetrical pulmonary lymphadenopathy, recommendations for or against a lymph node biopsy were not made, due to insufficient evidence, either for or against a routine lymph node biopsy. The need for further close clinical observation is emphasized in all patients whose biopsy was postponed.

In patients with suspected sarcoidosis, with hilar/mediastinal lymphadenopathy, in whom lymph node sampling is planned, endobronchial ultrasound (EBUS) bronchoscopy instead of mediastinoscopy is suggested as the first diagnostic tool (conditional recommendation, evidence of very low quality).

\section{Screening for extrapulmonary sarcoidosis}

In all patients diagnosed with sarcoidosis, screening for extrapulmonary lesions is suggested. Initial ophthalmological examination in all patients (regardless of the presence of ocular symptoms) is suggested as screening for ocular sarcoidosis (conditional recommendation, evidence of very low quality).

In the panel of initial laboratory tests, it is suggested that the concentration of serum creat-

Address for correspondence: Magdalena M Martusewicz-Boros, $3^{\text {rd }}$ Lung Diseases and Oncology Department, National Tuberculosis and Lung Diseases Research Institute, Warsaw, Poland; e-mail: m.martusewicz@gmail.com 
inine for the assessment of kidney involvement is determined. Serum alkaline phosphatase should be always included in the list of laboratory examinations as the best marker of liver sarcoidosis (experts do not present their opinion for or against routine transaminases testing). Complete blood cell count as an initial screening for hematological abnormalities (conditional recommendations, evidence of very low quality) is suggested in all patients. The only one strong recommendation in the entire document concerns the examination of serum calcium levels for abnormal calcium metabolism, which is recommended to all patients with sarcoid diagnosis (including those who have no symptoms or other signs of hypercalcemia) (strong recommendation, evidence of very low quality).

If the assessment of vitamin D metabolism is considered necessary in a patient with sarcoidosis, for instance in patients with indications for supplementation therapy, testing of both: 25 and $1.25-\mathrm{OH}$ vitamin $\mathrm{D}$ is suggested (conditional recommendation, evidence of very low quality).

To all patients with sarcoidosis who do not have cardiac symptoms, a resting electrocardiography (ECG) is recommended as a screening test for cardiac involvement (conditional recommendation, evidence of very low quality). If the patient has no cardiac symptoms, it is suggested that routine baseline echocardiography (ECHO) or outpatient 24-hour Holter ECG monitoring (conditional recommendation, evidence of very low quality) is NOT performed. The panel of experts concluded that the final decision whether or not to perform ECHO and Holter ECG should be considered individually in each case (conditional recommendation, evidence of very low quality).

\section{Diagnostics in patients with sarcoidosis of suspected extrapulmonary disease}

In patients diagnosed with sarcoidosis who are suspected of cardiac involvement - cardiac magnetic resonance imaging (CMRI) is suggested as a test of choice rather than cardiac positron emission tomography (CPET) or ECHO to obtain both, diagnostic and prognostic information. In cases where an MRI examination of the heart is not possible (due to lack of access to the equipment or contraindications to the procedure), it is suggested that CPET (conditional recommendations, evidence of very low quality) is performed.

To patients diagnosed with sarcoidosis who are suspected of having pulmonary hypertension $(\mathrm{PH})$, an ECHO test is suggested (conditional recommendation, evidence of very low quality). The $\mathrm{PH}$ suspicion is made up of clinical symptoms, including exercise pain in the chest and/or fainting, shortened distance in a 6-minute walk test, desaturation during exercise, in addition, results of additional tests indicating reduced lung transfer factor for carbon monoxide $\left(\mathrm{T}_{\mathrm{L}, \mathrm{CO}}\right)$, enlarged pulmonary artery diameter relative to ascending aorta diameter assessed in chest computed tomography (CT), increased brain natriuretic peptide (BNP) and/or the presence of pulmonary fibrosis.

To definitively confirm or exclude $\mathrm{PH}$ in patients diagnosed with sarcoidosis in whom ECHO result is suggestive of $\mathrm{PH}$, right heart catheterization (RHC) is suggested (conditional recommendation, very low-quality evidence). In the absence of suggestive echocardiographic changes for $\mathrm{PH}$, individual indications for diagnostic RHC should be considered (recommendation based on best clinical practice).

\section{Critical remarks and conclusion}

The question whether a biopsy is necessary in all patients suspected of sarcoidosis has been discussed for many years among all experts in the field, and this discussion usually results in vast spectrum of diverse opinions. Although the majority admits that strongly suggestive clinical symptoms (i.e. Lofgren syndrome), in addition to clear and doubtless radiological signs of bilateral lymphadenopathy, firmly support the diagnosis, the recommendation does not give a "one-fits-all" answer to this question. Erythema nodosum is not a specific sign of sarcoidosis, and among a long list of potential causes, one can find lymphoma and tuberculosis - both possibly presenting with mediastinal lymphadenopathy. The value of proper evaluation of radiological signs has not been stressed in the document strongly enough. The recommendation to postpone a biopsy in a patient with bilateral hilar lymphadenopathy seems to be valid only in individuals with evidently predominant bilateral enlargement of hilar lymph nodes, and in case of atypical configuration (i.e. predominance of non-hilar lymphadenopathy or asymmetry), the decision of a biopsy should be sustained. The authors of the guidelines use the term "mediastinal lymphadenopathy" not assuming an attitude to the presence or absence of coexisting parenchymal disease. These axillary features may either increase or decrease the diagnostic anxiety. Clearly enough, we do not have much evidence to answer such a question. EBUS is recommended but the question arises whether the presence of few epithelioid cells in the cytological examination indeed allows for exclusion 
of cancer-related sarcoid-like reaction or lymphoma? How to secure the diagnosis of sarcoidosis in view of the evidence that many patients suffering from tuberculosis and presenting with lymphadenopathy show non-caseating granulomas in the biopsy? The necessity of close monitoring (clearly emphasized by the guidelines' authors) and education of patients about the possible symptoms suggestive of alternative and much more dangerous diseases, possibly hiding under the mask of sarcoidosis, is of special importance. To conclude, individual approach is a key issue. Doctor's experience in the field is also extremely important, therefore, the best solution is to set up reference centers for sarcoidosis in order to minimize the risk of improper diagnosis.

The document does not discuss the importance of biopsies from bronchi or transbronchial biopsies (forceps or cryobiopsies). Obtaining confirmation of sarcoid granuloma from more than one organ significantly increases the probability of a correct diagnosis. Bronchoalveolar lavage (BAL), still routinely used in many centers worldwide, and a potential value of flow cytometry in the diagnosis of sarcoidosis have not been discussed either.

The recommendation concerning screening examinations does not cover all routine tests that are usually performed in sarcoid patients. There are several unanswered questions like the following: are spirometry and $\mathrm{T}_{\mathrm{L}, \mathrm{CO}}$ necessary in all patients? Should abdominal ultrasonography be performed for screening of abdominal locations in all patients? What is the value of chest CT in the initial evaluation, and when should it be used for monitoring? And last but not least, have we forgotten about the assessment of disease activity? Is it still important? In which patients? What about biomarkers (ACE, sR-IL2, neopterin etc.) or imaging techniques like PET or scintigraphy? The list of unanswered questions is probably much longer, and problems may be different depending on the local conditions, related to disease population characteristics, health system resources and organization.

The authors' own experience is in line with the indication of the cMRI as the superior method in assessing the involvement of this organ, after confirming sarcoid changes in the pathomorphological examination of lymph nodes, the lung or other organs. MRI allows both morphological and functional assessment, providing it is conducted according to an adequate protocol. At this point, it is worth emphasizing that diagnostic success is not dependent on the possibility of performing the test, i.e. access to appropriate hardware and software, but primarily depends on the substantive preparation of the person who carries out and evaluates the test.

In conclusion, the ATS panel of experts adopted one strong recommendation regarding the evaluation of serum calcemia, 13 conditional recommendations and one recommendation resulting from clinical practice ("best practice statement”). The strength of the recommendation regarding the assessment of serum calcemia is of great practical importance as significant hypercalcemia may be a life-threatening condition. Therefore, such assessment should take place in every patient at the stage of initial diagnosis, as well as in further monitoring of disease course. One should always regard a biopsy confirmation of sarcoid granuloma as a diagnostic standard, considering not only mediastinal lymph nodes as a biopsy site but also other techniques like bronchial biopsy, transbronchial lung biopsy, and other easily accessible locations like peripheral lymph nodes. Only in patients with highly suggestive clinical symptoms and typical radiological features, the biopsy may be postponed under the condition that close monitoring of disease course may be secured. The lack of a recommendation regarding the necessity of performing lymph node biopsies in a patient with asymptomatic mediastinal lymphadenopathy reflects the lack of sufficiently valuable evidence to support its performance in all patients. Regression of changes in subsequent imaging exams and a stable radiological image over a 2-year period exempt from the obligation of biopsy. In a situation when we decide to discontinue a biopsy, it is necessary to inform the patient about the need of systematic monitoring of the disease course. The patient should receive information on the symptoms that may indicate the need for deepening and reevaluation of the diagnosis.

The authors agree with the position of ATS experts that there is an urgent need for higher quality evidence that would support clinical practice in the diagnosis of sarcoidosis, which would help to better understand and define the natural course of the disease. Nevertheless, the document published by ATS is a valuable proposition of the current standard of sarcoidosis diagnosis, based on an objective analysis and currently available data. Despite having ATS recommendations, local societies should undertake an effort of preparing similar documents, asking more detailed and specific questions that would consider local conditions. 


\section{Conflict of interest}

None declared.

\section{References:}

1. Statement on sarcoidosis. Joint Statement of the American Thoracic Society (ATS), the European Respiratory Society (ERS) and the World Association of Sarcoidosis and Other
Granulomatous Disorders (WASOG) adopted by the ATS Board of Directors and by the ERS Executive Committee, February 1999. Am J Respir Crit Care Med. 1999; 160(2): 736-755, doi: 10.1164/ajrccm.160.2.ats4-99, indexed in Pubmed: 10430755.

2. Crouser ED, Maier LA, Wilson KC, et al. Diagnosis and Detection of Sarcoidosis. An Official American Thoracic Society Clinical Practice Guideline. Am J Respir Crit Care Med. 2020; 201(8): e26-e51, doi: 10.1164/rccm.202002-0251ST, indexed in Pubmed: $\underline{32293205}$. 